PROCEEDINGS OF THE

AMERICAN MATHEMATICAL SOCIETY

Volume 136, Number 9, September 2008, Pages 3263-3270

S 0002-9939(08)09429-X

Article electronically published on April 23, 2008

\title{
ALMOST POSITIVE CURVATURE ON THE GROMOLL-MEYER SPHERE
}

\author{
J.-H. ESCHENBURG AND M. KERIN \\ (Communicated by Jon G. Wolfson)
}

\begin{abstract}
Gromoll and Meyer have represented a certain exotic 7-sphere $\Sigma^{7}$ as a biquotient of the Lie group $G=S p(2)$. We show for a 2-parameter family of left invariant metrics on $G$ that the induced metric on $\Sigma^{7}$ has strictly positive sectional curvature at all points outside four subvarieties of codimension $\geq 1$ which we describe explicitly.
\end{abstract}

\section{INTRODUCTION}

Let $G=S p(2)$ be the Lie group of unitary quaternionic $2 \times 2$-matrices. Consider the subgroup $U \subset G \times G$,

$$
U=\left\{\left(\left({ }^{q}{ }_{1}\right),\left({ }^{q}{ }_{q}\right)\right) ; q \in S p(1)\right\},
$$

which acts on $G$ by left and right translations. D. Gromoll and W. Meyer [5] have shown that this action is free and that the orbit space $M=G / U$ is a smooth manifold which is an exotic 7-sphere (homeomorphic but not diffeomorphic to the standard 7 -sphere). If $G$ is equipped with a Riemannian metric of nonnegative sectional curvature whose isometry group contains $U$, then by O'Neill's formula 1 the orbit space $M=G / U$ inherits a Riemannian metric of nonnegative sectional curvature. Thus starting with the bi-invariant metric on $G$, Gromoll and Meyer constructed a metric of nonnegative sectional curvature on the exotic sphere $M$. In fact the curvature is strictly positive on some nonempty open subset of $M$. However, as was observed by F. Wilhelm [7, there is also an open subset with zero curvature planes in the tangent space of each of its points. But Wilhelm constructed another $U$-invariant metric on $S p(2)$ (neither left nor right invariant) for which the curvature of $M$ is strictly positive outside a subset of measure zero in $M$ ("almost positive curvature"). In [4 the same fact was claimed for a much simpler and left invariant metric on $S p(2)$; however, as was pointed out by the second author, the proof contains a serious mistake (see Remark 3 at the end of the present paper). The purpose of our paper is to correct this error. In fact we prove the following result, some ideas of which go back to [3] (see Theorem 4.6 for details):

Theorem 1.1. There is a left invariant and a $U$-invariant metric on $G=S p(2)$ such that the induced metric on $M=G / U$ has strictly positive curvature outside a

Received by the editors April 30, 2007.

2000 Mathematics Subject Classification. Primary 53C20, 53C30.

Key words and phrases. Biquotients, Lie groups, left invariant metrics, quaternions.

The second author would like to thank the University of Pennsylvania for financial support.

(C)2008 American Mathematical Society 
finite union of subvarieties of codimension $\geq 1$. The zero curvature set $Z \subset M$ can be explicitly determined.

\section{Cheeger metrics on Lie Groups}

On each Riemannian manifold, let us denote

$$
\begin{aligned}
\kappa(X, Y) & =\langle R(X, Y) Y, X\rangle, \\
\sec (X, Y) & =\kappa(X, Y) /|X \wedge Y|^{2}
\end{aligned}
$$

for any two tangent vectors $X, Y$; the second expression is the sectional curvature of the plane $\sigma$ spanned by $X, Y$.

Let $G$ be a Lie group with a left invariant metric $\langle$,$\rangle of nonnegative sectional$ curvature. Suppose that the metric is also right invariant with respect to a compact subgroup $K \subset G$, hence the induced metric on $K$ is bi-invariant. The Lie algebras of $G$ and $K$ will be denoted $\mathfrak{g}$ and $\mathfrak{k}$. We may contract the metric on $G$ in the direction of the $K$-cosets by viewing $G$ as the homogeneous space $(G \times K) / \Delta K$ (where $\Delta K=\{(k, k) ; k \in K\})$ and choosing the metric induced from the Riemannian product metric on $G \times s K$ (Cheeger contraction; cf. 2, 11) where $s K$ is $K$ with a metric scaled by $s>0$. A vector $\left(X, X^{\prime}\right) \in \mathfrak{g} \times \mathfrak{k}$ is perpendicular to the $\Delta K$-orbit ("horizontal") iff $X+s X^{\prime} \perp \mathfrak{k}$, i.e. $X^{\prime}=-s^{-1} X_{\mathfrak{k}}$ where $X_{\mathfrak{k}}$ is the $\mathfrak{k}$-projection of $X$. Using the Riemannian submersion $G \times K \rightarrow G,(g, k) \mapsto g k^{-1}$, a horizontal vector $\left(X,-s^{-1} X_{\mathfrak{k}}\right) \in \mathfrak{g} \times \mathfrak{k}$ is mapped onto $X+s^{-1} X_{\mathfrak{k}}=X_{\perp}+\left(1+s^{-1}\right) X_{\mathfrak{k}} \in \mathfrak{g}$ where $X_{\perp}=X-X_{\mathfrak{k}} \in \mathfrak{k}^{\perp}$. Vice versa, the horizontal lift of $X=X_{\perp}+X_{\mathfrak{k}} \in \mathfrak{g}$ is the horizontal vector

$$
\begin{aligned}
\widehat{X} & =\left(\tilde{X},-s^{-1} \tilde{X}_{\mathfrak{k}}\right), \quad \text { where } \\
\tilde{X} & =X_{\perp}+\frac{s}{s+1} X_{\mathfrak{k}} .
\end{aligned}
$$

Thus the new (left invariant) metric is

$$
\begin{aligned}
\langle X, Y\rangle_{1} & =\langle\widehat{X}, \widehat{Y}\rangle \\
& =\langle\tilde{X}, \tilde{Y}\rangle+s\left\langle s^{-1} \tilde{X}_{\mathfrak{k}}, s^{-1} \tilde{Y}_{\mathfrak{k}}\right\rangle \\
& =\langle\tilde{X}, \tilde{Y}\rangle+s^{-1}\left\langle\tilde{X}_{\mathfrak{k}}, \tilde{Y}_{\mathfrak{k}}\right\rangle \\
& =\left\langle\tilde{X} \tilde{Y}_{\perp}\right\rangle+s^{-1}(s+1)\left\langle\tilde{X}_{\mathfrak{k}}, \tilde{Y}_{\mathfrak{k}}\right\rangle \\
& =\left\langle X_{\perp}, Y_{\perp}\right\rangle+s(s+1)^{-1}\left\langle X_{\mathfrak{k}}, Y_{\mathfrak{k}}\right\rangle \\
& =\langle\tilde{X}, Y\rangle .
\end{aligned}
$$

For the curvature terms we have

$$
\kappa(\widehat{X}, \widehat{Y})=\kappa(\tilde{X}, \tilde{Y})+s^{-3} \kappa\left(\tilde{X}_{\mathfrak{k}}, \tilde{Y}_{\mathfrak{k}}\right) .
$$

Since all terms are nonnegative, the left hand side vanishes if and only if both summands on the right are zero. Thus a plane $\sigma$ spanned by $X, Y \in \mathfrak{g}$ has zero curvature in the new metric, $\sec _{1}(\sigma)=0$, if and only if $\sec (\tilde{\sigma})=0$ and $\left[X_{\mathfrak{k}}, Y_{\mathfrak{k}}\right]=011$

Example 1. Suppose that the initial metric $\langle$,$\rangle on G$ is bi-invariant. Let $\mathfrak{g}=\mathfrak{k}+\mathfrak{p}$ be the orthogonal decomposition. Consider the above metric

$$
\langle X, Y\rangle_{1}=\left\langle X_{\mathfrak{p}}, Y_{\mathfrak{p}}\right\rangle+\tilde{s}\left\langle X_{\mathfrak{k}}, Y_{\mathfrak{k}}\right\rangle
$$

\footnotetext{
${ }^{1}$ The "if" statement is not obvious because of the nonnegative O'Neill term. However, in all our examples starting with a bi-invariant metric on some Lie group, the vanishing of the curvature implies that the O'Neill term also vanishes; see [3], pp. 29ff, equations (1) - (4) or [8], 6].
} 
with $\tilde{s}=\frac{s}{s+1}$. Then $\sec (\tilde{\sigma})=0 \Longleftrightarrow[\tilde{X}, \tilde{Y}]=0$, and hence $\sec _{1}(\sigma)=0 \Longleftrightarrow$

$$
[\tilde{X}, \tilde{Y}]=0, \quad\left[X_{\mathfrak{k}}, Y_{\mathfrak{k}}\right]=0 .
$$

If $(G, K)$ is a symmetric pair, i.e. the orthogonal complement $\mathfrak{p} \subset \mathfrak{g}$ satisfies $[\mathfrak{p}, \mathfrak{p}] \subset \mathfrak{k}$, then $\mid \tilde{X}, \tilde{Y}]_{\mathfrak{k}}=\left[\tilde{X}_{\mathfrak{k}}, \tilde{Y}_{\mathfrak{k}}\right]+\left[\tilde{X}_{\mathfrak{p}}, \tilde{Y}_{\mathfrak{p}}\right]$ and $\left.\mid \tilde{X}, \tilde{Y}\right]_{\mathfrak{p}}=\left[\tilde{X}_{\mathfrak{k}}, \tilde{Y}_{\mathfrak{p}}\right]+\left[\tilde{X}_{\mathfrak{p}}, \tilde{Y}_{\mathfrak{k}}\right]$; hence $\sec _{1}(\sigma)=0 \Longleftrightarrow$

$$
0=\left[X_{\mathfrak{k}}, Y_{\mathfrak{k}}\right]=\left[X_{\mathfrak{p}}, Y_{\mathfrak{p}}\right]=\left[X_{\mathfrak{k}}, Y_{\mathfrak{p}}\right]+\left[X_{\mathfrak{p}}, Y_{\mathfrak{k}}\right]=[X, Y] .
$$

Example 2. Let $G \supset K \supset H$ be a chain of subgroups and suppose that both $(G, K)$ and $(K, H)$ are symmetric pairs. Let $\mathfrak{g}=\mathfrak{k}+\mathfrak{p}$ and $\mathfrak{k}=\mathfrak{h}+\mathfrak{q}$ be the corresponding decompositions. Start with the metric $\langle,\rangle_{1}$ defined by Example 1, depending on a parameter $s>0$, and define the metric $\langle,\rangle_{2}$ by Cheeger contraction along $H$ (depending on a new parameter $t>0$ ) as in (2.3), where $K$ is replaced by $H$ and $\langle,\rangle_{1}$ takes the role of $\langle$,$\rangle :$

$$
\begin{aligned}
\langle X, Y\rangle_{2} & =\left\langle X_{\mathfrak{p}}, Y_{\mathfrak{p}}\right\rangle_{1}+\left\langle X_{\mathfrak{q}}, Y_{\mathfrak{q}}\right\rangle_{1}+\tilde{t}\left\langle X_{\mathfrak{h}}, Y_{\mathfrak{h}}\right\rangle_{1} \\
& =\left\langle X_{\mathfrak{p}}, Y_{\mathfrak{p}}\right\rangle+\tilde{s}\left\langle X_{\mathfrak{q}}, Y_{\mathfrak{q}}\right\rangle+\tilde{s} \tilde{t}\left\langle X_{\mathfrak{h}}, Y_{\mathfrak{h}}\right\rangle
\end{aligned}
$$

with $\tilde{t}=\frac{t}{t+1}$. Then $\sec _{2}(\sigma)=0 \Longleftrightarrow \sec _{1}(\tilde{\sigma})=0$ and $\left[\tilde{X}_{\mathfrak{h}}, \tilde{Y}_{\mathfrak{h}}\right]=0 \Longleftrightarrow$

$$
0=[\tilde{X}, \tilde{Y}]=\left[\tilde{X}_{\mathfrak{k}}, \tilde{Y}_{\mathfrak{k}}\right]=\left[X_{\mathfrak{p}}, Y_{\mathfrak{p}}\right]=\left[X_{\mathfrak{q}}, Y_{\mathfrak{q}}\right]=\left[X_{\mathfrak{h}}, Y_{\mathfrak{h}}\right],
$$

where $\tilde{X}=X_{\mathfrak{p}}+X_{\mathfrak{q}}+\frac{t}{t+1} X_{\mathfrak{h}}$ and $\tilde{Y}=Y_{\mathfrak{p}}+Y_{\mathfrak{q}}+\frac{t}{t+1} Y_{\mathfrak{h}}$, as in (2.2).

\section{Zero CuRVature Planes on $S p(2)$}

Let us consider the chain $G \supset K \supset H$ for $G=S p(2), K=S p(1) \times S p(1)$ and $H=\Delta S p(1)=\left\{\left({ }^{q}{ }_{q}\right) ; q \in S p(1)\right\}$. The pairs $(G, K)$ and $(K, H)$ are symmetric, corresponding to the rank-one symmetric spaces $S^{4}$ and $S^{3}$. We start with the bi-invariant trace metric $\langle X, Y\rangle=\operatorname{Re}$ trace $X^{*} Y=\operatorname{Re} \sum \overline{x_{i j}} y_{i j}$ on $\mathfrak{g}=\mathfrak{s p}(2)$, apply Cheeger contraction in the $K$-direction, and Cheeger-contract again in the $H$-direction, defining metrics $\langle,\rangle_{1}$ and $\langle,\rangle_{2}$ as in Example 2.

Since $G / K=S^{4}$ as well as $K / H=S^{3}$ and $H=S^{3}$ have positive curvature, the vanishing of the last three brackets in (2.8) means the linear dependence of the factors. In particular we may assume $Y_{\mathfrak{p}}=0$, i.e. $\tilde{Y}=\tilde{Y}_{\mathfrak{k}}=\left(\begin{array}{ll}y_{1} & \\ & y_{2}\end{array}\right)$.

Case 1. $X_{\mathfrak{p}}=0$, i.e. $\tilde{X}=\tilde{X}_{\mathfrak{k}}=\left(\begin{array}{ll}x_{1} & \\ & x_{2}\end{array}\right)$.

From $\left[\tilde{X}_{\mathfrak{k}}, \tilde{Y}_{\mathfrak{k}}\right]=0$ we obtain that the imaginary quaternions $x_{1}, y_{1}$ as well as $x_{2}, y_{2}$ are linearly dependent. Moreover, from $\left[X_{\mathfrak{q}}, Y_{\mathfrak{q}}\right]=\left[X_{\mathfrak{h}}, Y_{\mathfrak{h}}\right]=0$ we also see that $x_{1} \pm x_{2}$ and $y_{1} \pm y_{2}$ are linearly dependent. Putting $y=y_{1}$, we may assume

$$
\tilde{Y}=\left(\begin{array}{cc}
y & \\
& 0
\end{array}\right), \quad \tilde{X}=\left(\begin{array}{ll}
0 & \\
& y
\end{array}\right) .
$$

Case 2. $X_{\mathfrak{p}} \neq 0$, i.e. $X=\left(\begin{array}{cc}x_{1} & -\bar{x} \\ x & x_{2}\end{array}\right)$ with $x \neq 0$.

Then $0=[\tilde{X}, \tilde{Y}]_{\mathfrak{p}}=\left[X_{\mathfrak{p}}, \tilde{Y}\right] \Longleftrightarrow y_{2}=x y x^{-1}$ for $y:=y_{1}$, and $0=[\tilde{X}, \tilde{Y}]_{\mathfrak{k}}=$ $\left[\tilde{X}_{\mathfrak{k}}, \tilde{Y}_{\mathfrak{k}}\right] \Longleftrightarrow x_{1}=\alpha y_{1}, x_{2}=\beta y_{2}$ for real numbers $\alpha, \beta$; hence

$$
\tilde{Y}=\left(\begin{array}{cc}
y & \\
& x y x^{-1}
\end{array}\right), \quad \tilde{X}=\left(\begin{array}{cc}
\alpha y & -\bar{x} \\
x & -\alpha x y x^{-1}
\end{array}\right),
$$


where $x, y \in \mathbb{H}, y$ is imaginary and $\alpha \in \mathbb{R}$. We have $\beta=-\alpha$ since we require $\langle\tilde{X}, \tilde{Y}\rangle=0$.

Case 2a. $\alpha=0$; hence

$$
\tilde{Y}=\left(\begin{array}{ll}
y & \\
& x y x^{-1}
\end{array}\right), \quad \tilde{X}=\left(\begin{array}{ll} 
& -\bar{x} \\
x &
\end{array}\right) .
$$

Case 2b. $\alpha \neq 0$; hence (without loss of generality) $\alpha=1$.

Then $\left[X_{\mathfrak{h}}, Y_{\mathfrak{h}}\right]=0$ iff $y+x y x^{-1}$ and $y-x y x^{-1}$ are proportional, which means $x y x^{-1}=\beta y$. Comparing the norms on both sides we get

$$
x y x^{-1}= \pm y
$$

and

$$
\tilde{Y}=Y_{ \pm}=\left(\begin{array}{cc}
y & \\
& \pm y
\end{array}\right), \quad \tilde{X}=X_{ \pm}=\left(\begin{array}{cc}
y & -\bar{x} \\
x & \mp y
\end{array}\right) .
$$

Lemma 3.1. The zero curvature planes in $\mathfrak{g}=T_{e} G$ for $G=S p(2)$ and the metric $\langle,\rangle_{2}$ are spanned by $X, Y \in \mathfrak{g}$ with $\tilde{X}, \tilde{Y}$ given by either (3.1) or (3.3) or (3.5).

\section{The Gromoll-Meyer sphere}

The metric $\langle,\rangle_{2}$ on $G=S p(2)$ is invariant under the action of $U$ (cf. (1.1)) and hence it induces a metric on the orbit space $M=G / U$. Consider any

$$
g=\left(\begin{array}{ll}
a & b \\
c & d
\end{array}\right) \in G
$$

Since $g$ is unitary, the rows and columns are unit vectors, in particular

$$
|a|^{2}+|b|^{2}=1 \text {. }
$$

The vertical space at $g$ of the submersion $\pi: G \rightarrow G / U$ is $T_{g}(U . g)=g V_{g}$ with $V_{g}=\left\{v_{g} ; v \in \operatorname{Im} \mathbb{H}\right\}$ where

$$
v_{g}=g^{-1}\left(\begin{array}{ll}
v & 0 \\
0 & 0
\end{array}\right) g-\left(\begin{array}{cc}
v & 0 \\
0 & v
\end{array}\right)=\left(\begin{array}{cc}
\bar{a} v a-v & \bar{a} v b \\
\bar{b} v a & \bar{b} v b-v
\end{array}\right) .
$$

Thus according to (2.3), a vector $g X \in T_{g} G$ is horizontal for $\pi$ iff

$$
0=\left\langle X, v_{g}\right\rangle_{2}=\left\langle\tilde{X}, v_{g}\right\rangle_{1}
$$

for all $v \in \operatorname{Im} \mathbb{H}$. Note that $\left\langle\tilde{X}, v_{g}\right\rangle_{1}$ is just a multiple of $\left\langle\tilde{X}, v_{g}\right\rangle$ if one of the components of $\tilde{X}=\tilde{X}_{\mathfrak{p}}+\tilde{X}_{\mathfrak{k}}$ are zero. Now we discuss which of the zero curvature planes in $G=S p(2)$ (see Lemma 3.1) can be horizontal at any $g \in G$. By a slight abuse of language, a plane $\tilde{\sigma}$ spanned by $\tilde{X}, \tilde{Y} \in \mathfrak{g}$ will be called horizontal at $g$ if

$$
\left\langle\tilde{X}, v_{g}\right\rangle_{1}=\left\langle\tilde{Y}, v_{g}\right\rangle_{1}=0
$$

for all $v \in \operatorname{Im} \mathbb{H}$.

Case 1.

Lemma 4.1. A plane of type (3.1) is nowhere horizontal.

Proof. $\left\langle\tilde{Y}, v_{g}\right\rangle=\langle y, \bar{a} v a-v\rangle=\langle a y \bar{a}-y, v\rangle$ vanishes for all $v \in \operatorname{Im} \mathbb{H}$ iff $y=a y \bar{a}$, and likewise $\left\langle\tilde{X}, v_{g}\right\rangle$ vanishes for all $v$ iff $y=b y \bar{b}$. But this implies $|a|=|b|=1$ in contradiction to (4.2). 


\section{Case 2a.}

Lemma 4.2. If a plane of type (3.3) is horizontal at $g$, then either $a=0$ or $b=0$ or

$$
\operatorname{det}\left(I-\operatorname{Ad}\left(a^{-1}\right)-\operatorname{Ad}\left(b^{-1}\right)\right)=0 .
$$

Proof. The matrix $\tilde{X}$ is horizontal at $g$ if and only if

$$
0=\left\langle\tilde{X}, v_{g}\right\rangle=2\langle x, \bar{b} v a\rangle=2\langle b x \bar{a}, v\rangle
$$

for all $v \in \operatorname{Im} \mathbb{H}$. This is equivalent to $b x \bar{a} \in \mathbb{R}$. Hence, either $a=0$ or $b=0$ or $b x=r a$ for some nonzero $r \in \mathbb{R}$. In the latter case we have, in particular

$$
\begin{aligned}
\operatorname{Ad}(b x) & =\operatorname{Ad}(a), \\
\operatorname{Ad}(x) & =\operatorname{Ad}\left(b^{-1}\right) \operatorname{Ad}(a),
\end{aligned}
$$

provided that $b \neq 0$. On the other hand, the matrix $\tilde{Y}$ is horizontal at $g$ if and only if

$$
0=\left\langle\tilde{Y}, v_{g}\right\rangle=\left\langle|a|^{2} \operatorname{Ad}(a) y-y+|b|^{2} \operatorname{Ad}(b x) y-\operatorname{Ad}(x) y, v\right\rangle
$$

for all $v \in \operatorname{Im} \mathbb{H}$. Since $y \in \operatorname{Im} \mathbb{H}$, this means

$$
\begin{aligned}
0 & =|a|^{2} \operatorname{Ad}(a) y+|b|^{2} \operatorname{Ad}(b x) y-y-\operatorname{Ad}(x) y \\
& \stackrel{4.8}{=} \operatorname{Ad}(a) y-y-\operatorname{Ad}(x) y \\
& \stackrel{4.9}{=} \operatorname{Ad}(a) y-y-\operatorname{Ad}\left(b^{-1}\right) \operatorname{Ad}(a) y,
\end{aligned}
$$

where we have also used $|a|^{2}+|b|^{2}=1$ (4.2). If $a \neq 0$, we obtain from the last equality

and in particular

$$
\operatorname{Ad}(a) y \in \operatorname{ker}\left(I-\operatorname{Ad}\left(a^{-1}\right)-\operatorname{Ad}\left(b^{-1}\right)\right)
$$

$$
\operatorname{det}\left(I-\operatorname{Ad}\left(a^{-1}\right)-\operatorname{Ad}\left(b^{-1}\right)\right)=0 .
$$

Lemma 4.3. There exists a plane of type (3.3) which is horizontal at $g$ if and only if either (4.6) holds or

$$
a=0, \quad|\operatorname{Im} b| \geq \frac{1}{2} \text { or } \quad b=0, \quad|\operatorname{Im} a| \geq \frac{1}{2} .
$$

Proof. Suppose first that $a, b \neq 0$. If (4.6) is satisfied, there is a nonzero $w \in$ $\operatorname{ker}\left(I-\operatorname{Ad}\left(a^{-1}\right)-\operatorname{Ad}\left(b^{-1}\right)\right)$. Then defining $y=\operatorname{Ad}\left(a^{-1}\right) w$ and $x=b^{-1} a$, we obtain a horizontal plane of type (3.3) at $g$. The converse conclusion was done before.

Now suppose $b=0$. Then $|a|=1$ and equation (4.11) becomes

$$
\operatorname{Ad}(a) y-y=\operatorname{Ad}(x) y \text {. }
$$

Geometrically, this equality means that $\operatorname{Ad}(a)$ rotates $y$ by the angle $\frac{\pi}{3}$ (the three vectors $\operatorname{Ad}(a) y, y$, and $\operatorname{Ad}(x) y$ form the sides of an equilateral triangle). Hence (4.13) has a solution $(x, y)$ if and only if the rotation angle of the rotation $\operatorname{Ad}(a)$ is $\geq \frac{\pi}{3}$. This in turn is equivalent to $\varangle(a, 1) \geq \frac{\pi}{6}$, i.e. $|\operatorname{Im} a| \geq \frac{1}{2}$. Inserting the solution $(x, y)$ into (3.3) defines a horizontal plane of type (3.3). The case $a=0$ is similar. 


\section{Case 2b.}

Lemma 4.4. If a plane of type (3.5) is horizontal at $g$, then

$$
|a|=|b|=1 / \sqrt{2}
$$

and $w:=\operatorname{Im} a^{-1} b$ satisfies

$$
\left\langle w-2 a^{-1} w a, w\right\rangle=0
$$

Proof.

$$
\begin{aligned}
& \left\langle v_{g}, Y_{+}\right\rangle=\langle\bar{a} v a+\bar{b} v b-2 v, y\rangle=\langle v, a y \bar{a}+b y \bar{b}-2 y\rangle, \\
& \left\langle v_{g}, Y_{-}\right\rangle=\langle\bar{a} v a-\bar{b} v b, y\rangle=\langle v, a y \bar{a}-b y \bar{b}\rangle .
\end{aligned}
$$

Thus $\left\langle\tilde{Y}, V_{g}\right\rangle=0$ iff one of the following equations holds:

$$
\begin{aligned}
& a y \bar{a}+b y \bar{b}=2 y, \\
& a y \bar{a}-b y \bar{b}=0 .
\end{aligned}
$$

The first of these equations is impossible by the triangle inequality together with (4.2):

$$
|a y \bar{a}+b y \bar{b}| \leq|a y \bar{a}|+|b y \bar{b}| \leq\left(|a|^{2}+|b|^{2}\right)|y|=|y|<|2 y| .
$$

Thus we are left with the second equation,

$$
a y \bar{a}=b y \bar{b},
$$

which implies $|a|=|b|$.

Note that we have also shown that $Y_{+}$cannot be horizontal. Thus we need only consider $\tilde{X}=X_{-}$and $\tilde{Y}=Y_{-}$in (3.5), and

$$
x y x^{-1}=-y,
$$

which means that $x$ is imaginary and nonzero with $x \perp y$.

Now let $\tilde{X}, \tilde{Y}$ be as above spanning $\tilde{\sigma}$. By (3.5) we have

$$
\tilde{Y}=\left(\begin{array}{cc}
y & \\
& -y
\end{array}\right), \quad \tilde{X}=\left(\begin{array}{cc}
y & x \\
x & y
\end{array}\right)
$$

with $y \perp x \in \operatorname{Im} \mathbb{H}$. Thus according to (2.5) we get for all $v \in \operatorname{Im} \mathbb{H}$

$$
\begin{aligned}
0=\left\langle\tilde{X}, v_{g}\right\rangle_{1} & =2\langle x, \bar{b} v a\rangle+\tilde{s}\langle y, \bar{a} v a+\bar{b} v b-2 v\rangle \\
& =2\langle b x \bar{a}, v\rangle+\tilde{s}\langle a y \bar{a}+b y \bar{b}-2 y, v\rangle \\
& =\left\langle b x a^{-1}+\tilde{s}\left(a y a^{-1}-2 y\right), v\right\rangle,
\end{aligned}
$$

where we have used $2 \bar{a}=a^{-1}$ and $a y \bar{a}=b y \bar{b}=\frac{1}{2} a y a^{-1}$ from (4.14) and (4.18). Putting $p=a^{-1} b / \tilde{s}$, we obtain

$$
\operatorname{Im} a p x a^{-1}=2 y-a y a^{-1} .
$$

From $a y a^{-1}=b y b^{-1}$ we see $y p=p y$; thus $p \in \mathbb{C}_{y}:=\mathbb{R}+\mathbb{R} y$ and the left multiplication with $p$ preserves $\mathbb{C}_{y}$ and $\mathbb{C}_{y}^{\perp}$. By (4.19) we have $x \in \mathbb{C}_{y}^{\perp}$ and therefore $p x \in \mathbb{C}_{y}^{\perp}$. Conjugating (4.22) by $a^{-1}$ we obtain

$$
\begin{aligned}
2 a^{-1} y a-y & =\operatorname{Im}(p x) \perp y, \\
\left\langle 2 a^{-1} y a-y, y\right\rangle & =0 .
\end{aligned}
$$

Since $w=\operatorname{Im} \tilde{s} p \in \mathbb{C}_{y}$ is a multiple of $y$, we may replace $y$ by $w$ in equation (4.24) and obtain 4.15). 
Remark 1.

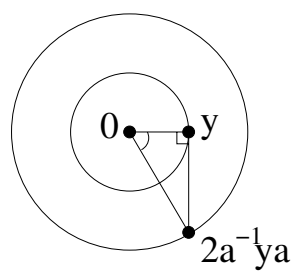

Geometrically, (4.24) means that the angle between $y$ and $a^{-1} y a$ is $\pi / 3=60^{\circ}$. Thus the rotation angle of $\operatorname{Ad}\left(a^{-1}\right)$ (and of $\operatorname{Ad}\left(b^{-1}\right)$; see (4.18) ) must be $\geq \pi / 3$; hence $\varangle(1, a) \geq \pi / 6$, or in other words,

$$
\frac{|\operatorname{Im} a|}{|a|} \geq \frac{1}{2}
$$

Lemma 4.5. Suppose that $a, b \in \mathbb{H}$ satisfy (4.14), (4.15) and (4.25). Then there exists a horizontal plane of type (3.5) at $g=\left(\begin{array}{ll}a & b \\ c & d\end{array}\right)$.

Proof. First suppose that $\tilde{p}=a^{-1} b=\tilde{s} p$ is real which in view of (4.14) means $a= \pm b$. By (4.25), the rotation angle of $\operatorname{Ad}\left(a^{-1}\right)$ is $\geq \pi / 3$; hence there exists a nonzero $y \in \operatorname{Im} \mathbb{H}$ which is rotated precisely by the angle $\pi / 3$ and thus satisfies (4.24). Put $x=2 a^{-1} y a-y \perp y$ and define $\tilde{X}, \tilde{Y}$ as in (4.20). This matrix pair is of type (3.5), and it is perpendicular to $V_{g}$ by (4.17) and (4.21).

Now suppose that $w=\operatorname{Im} \tilde{p} \neq 0$; in this case (4.15) implies (4.25). Then we choose $y=w$ and $x=\operatorname{Im}\left(p^{-1}\left(2 a^{-1} w a-w\right)\right)$; compare (4.23). Since $w-2 a^{-1} w a \in$ $\mathbb{C}_{y}^{\perp}$ (it is imaginary and perpendicular to $w=y$ ), we also have $p^{-1}\left(w-2 a^{-1} w a\right) \in$ $\mathbb{C}_{y}^{\perp}$; hence $x \perp y$ and thus $x y x^{-1}=-y$. Defining matrices $\tilde{X}, \tilde{Y}$ using (4.20), these are of type (3.5) and perpendicular to $V_{g}$ by (4.17) and (4.21).

Remark 2. Clearly, the relations (4.6), (4.12), (4.14), (4.15) and (4.25) must be invariant under the action of $U$. In fact, if $u=\left(\left({ }^{q}{ }_{1}\right),\left({ }^{q}{ }_{q}\right)\right)$, we have $u \cdot g=\tilde{g}=$ $\left(\begin{array}{ll}\tilde{a} & \tilde{b} \\ \tilde{c} & \tilde{d}\end{array}\right)$ with $\tilde{a}=q a q^{-1}$ and $\tilde{b}=q b q^{-1}$.

Now we have proved the following.

Theorem 4.6. Let $G=S p(2)$ with the left invariant metric (2.7) and $U \subset G \times G$ defined by (1.1). The orbit space $M=G / U$ inherits a Riemannian metric such that the canonical projection $\pi: G \rightarrow M$ is a Riemannian submersion. Let

$$
Z=\left\{p \in M ; \exists \sigma \subset T_{p} M: \sec (\sigma)=0\right\} .
$$

Then $Z=Z_{1} \cup Z_{2} \cup Z_{3} \cup Z_{4}$ where

$$
\begin{aligned}
& \pi^{-1} Z_{1}=\left\{\left(\begin{array}{ll}
a & b \\
c & d
\end{array}\right) ; a, b \neq 0, \operatorname{det}\left(I-\operatorname{Ad}\left(a^{-1}\right)-\operatorname{Ad}\left(b^{-1}\right)\right)=0\right\}, \\
& \pi^{-1} Z_{2}=\left\{\left(\begin{array}{ll}
a & b \\
c & d
\end{array}\right) ;|a|=|b|, w:=\operatorname{Im} a^{-1} b \perp w-2 a^{-1} w a,|\operatorname{Im} a| \geq|a| / 2\right\}, \\
& \pi^{-1} Z_{3}=\left\{\left(\begin{array}{ll}
a & b \\
c & d
\end{array}\right) ; b=c=0,|\operatorname{Im} a| \geq 1 / 2\right\}, \\
& \pi^{-1} Z_{4}=\left\{\left(\begin{array}{ll}
a & b \\
c & d
\end{array}\right) ; a=d=0,|\operatorname{Im} b| \geq 1 / 2\right\},
\end{aligned}
$$

where all matrices $\left(\begin{array}{ll}a & b \\ c & d\end{array}\right)$ are supposed to belong to $S p(2)$.

Remark 3. The mistake in [4] is in the third line of the proof of the theorem, page 1166. The computation of $\left\langle v_{g}, X\right\rangle$ holds only for $X \in \mathfrak{k}$, but $X$ may have a nonzero $\mathfrak{p}$-component as well. Thus the matrix $X$ in (4), p. 1166, is too special and must be replaced with the more general $X=\left(\begin{array}{cc}r y & -\bar{x} \\ x & -r x y x^{-1}\end{array}\right)$ for arbitrary $r \in \mathbb{R}$, and 
instead of (5) $\operatorname{Im}(b x \bar{a})=0$ we obtain $\left(5^{\prime}\right) \operatorname{Im}(b x \bar{a})=r(y-a y \bar{a})$, while equation (6) $\left(a y \bar{a}-y+b x y x^{-1} \bar{b}-x y x^{-1}=0\right)$ remains unchanged. We have 15 variables, $(a, b) \in S^{7}, x \in \mathbb{H}, y \in \operatorname{Im}(\mathbb{H}), r \in \mathbb{R}$, with 2 arbitrary real constants (the lengths of $x$ and $y$ ), and 6 constraint equations $\left(5^{\prime}\right)$ and (6) which reduce the number of free variables to 7 . Thus the solution set is likely to project onto a subset with positive measure in the $(a, b)$-space $S^{7}$; this would imply that the metric considered in [4] fails to have almost positive curvature.

\section{REFERENCES}

[1] A.L. Besse: Einstein Manifolds, Springer, Berlin, 1987. MR867684 (88f:53087)

[2] J. Cheeger: Some examples of manifolds of nonnegative curvature, J. Diff. Geom. 8 (1973), 623 - 628. MR0341334(49:6085)

[3] J.-H. Eschenburg: Freie isometrische Aktionen auf kompakten Lie-Gruppen mit positiv gekrümmten Orbiträumen, Schriftenreihe Math. Inst. Univ. Münster (2) 32, Universität Münster, Mathematisches Institut, Münster (1984). MR758252 (86a:53045)

[4] J.-H. Eschenburg: Almost positive curvature on the Gromoll-Meyer 7-sphere, Proc. Amer. Math. Soc. 130, No. 4 (2002), 1165 - 1167. MR1873792(2002i:53045)

[5] D. Gromoll, W.T. Meyer: An exotic sphere with nonnegative sectional curvature, Ann. of Math. 100 (1974), 401 - 406. MR0375151 (51:11347)

[6] K. Tapp: Flats in Riemannian submersions from Lie groups, preprint (2007), DG0703389.

[7] F. Wilhelm: An exotic sphere with positive curvature almost everywhere, J. Geom. Anal. 11 (2001), 519 - 560. MR1857856 (2002f:53056)

[8] B. Wilking: Manifolds with positive sectional curvature almost everywhere, Invent. Math. 148 (2002), 117-141. MR1892845 (2003a:53049)

Institut für Mathematik, Universität Augsburg, D-86135 Augsburg, Germany

E-mail address: eschenburg@math.uni-augsburg.de

Department of Mathematics, University of Pennsylvania, 209 S 33rd St., PhiladelPhia, Pennsylvania 19104

E-mail address: mkerin@math.upenn.edu 